\title{
Physicalische Beschreibung der Canarischen Inseln de von Buch y la terminología de la traducción al español de Delgado Luis ${ }^{1}$
}

\author{
Physicalische Beschreibung der Canarischen Inseln by von Buch and its \\ terminology in its translation into Spanish by Delgado Luis
}

\author{
Ana María Monterde Rey² · https://orcid.org/0000-0002-0275-8216 \\ Universidad de Las Palmas de Gran Canaria \\ C/ Pérez del Toro, 1 (35003-Las Palmas de Gran Canaria)
}

\section{ABSTRACt}

The goal of this paper is to analyze the terminology of the only full translation of the book Physicalische Beschreibung der Canarischen Inseln by Buch carried out by Delgado Luis (von Buch, 1999). In order to achieve this goal, we perform the extraction of all the terms of the book by using Trados SDL MultiTerm Extract and creating a terminological data base. Analyzing this data base, we study the terms used in the translation: terminological density, methods of term formation and strategies of translation. With all this, we determine de terminogical adequacy of the translated book contributing with a new knowledge of this importart text.

$$
\text { Keywords: terminography, terminology, von Buch. }
$$

\section{RESUMEN}

El objeto de este artículo es analizar la terminología de la única traducción completa al español de Physicalische Beschreibung der Canarischen Inseln de von Buch efectuada por Delgado Luis (von Buch, 1999). Con este fin, efectuamos un vaciado terminológico de la obra con SDL MultiTerm Extract de Trados y creamos una base datos con MultiTerm. A partir de ella, estudiamos los términos empleados en la traducción: densidad terminológica, métodos de formación de términos y estrategias de traducción. Con ello determinamos la adecuación terminológica de la obra traducida aportando, así, un nuevo conocimiento de esta importante obra.

Palabras clave: terminografía, terminología, von Buch.

\section{Introducción}

Las Islas Canarias han sido desde su conquista en el siglo XV un lugar de estudio para naturalistas europeos. A ellas llegaron numerosos viajeros y científicos atraídos por su geología, su climatología y su mundo aborigen (Hernández González, 2011: 11). Son varias las obras naturalistas sobre estas islas que tuvieron repercusión en el mundo de la geología en distintos siglos. El mayor avance en este campo tuvo lugar en el siglo XIX. En este

Este trabajo supone una ampliación de nuestro capítulo que se puede leer en Monterde Rey y Sarmiento Pérez y complementa otro sobre la visión predarwinista de Buch en relación con la flora del Teide (Sarmiento Pérez, 2018).

Corresponding author·Email: anamaria.monterde@ulpgc.es 
siglo destaca una obra publicada por Leopold von Buch en 1825: Physicalische Beschreibung der Canarischen Inseln.

Nuestro propósito es analizar la terminología de la única traducción completa al español de esta importante publicación científica geológica efectuada por Delgado Luis (von Buch, 1999), estudio que nunca antes se ha llevado a cabo a pesar de la gran importancia de esta obra. Para ello, primero vamos a encuadrar la obra históricamente en las expediciones científicas decimonónicas y justificaremos su elección. Después de resaltar la relevancia del texto original y efectuar un pequeño resumen de su contenido, pasaremos a recrearnos en su traducción y su repercusión en las Canarias. Al tratarse de una traducción científica caracterizada por el uso de términos, reflexionaremos sobre la traducción de obras científicas en el siglo XIX y la traducción de su terminología. Finalmente entraremos en el estudio estrictamente terminológico. Con este fin, ejecutaremos un vaciado terminológico de la obra con herramientas informáticas y, a partir de él, estudiaremos los términos empleados en la traducción: densidad terminológica, métodos de formación de términos y estrategias de traducción. Con ello determinaremos la adecuación terminológica de la obra traducida y aportaremos un nuevo conocimiento de este texto.

\section{La exploración naturalista de las Islas Canarias en los siglos XVIII y XIX}

Desde su conquista en 1492, las Islas Canarias han sido un enlace entre continentes. Al principio (Oliver Frade y Relancio Menéndez, 2007: 11) su interés estaba ligado a la navegación marítima (eran tierras de referencia para los viajes por el Atlántico) y al reabastecimiento de las tripulaciones para los viajes entre continentes. Estas escalas hicieron que poco a poco se fuera descubriendo el interés científico de las islas, que cuenta con numerosas especies endémicas, dado su aislamiento. Así ya en el siglo XVIII las islas se convirtieron en un laboratorio de pruebas para los naturalistas que formaban parte de las expediciones científicas transoceánicas. En sus visitas, fueron numerosos los investigadores que dejaron guías, libros, relatos de viaje, memorias, etc.

En este siglo destaca la estancia de Alexander von Humboldt en Tenerife en junio de 1799. Su descripción de la isla animó a otros viajeros y naturalistas a emprender viajes de exploración. La segunda isla más visitada fue Gran Canaria, seguida por La Palma y, a continuación, Fuerteventura y Lanzarote, principalmente por viajeros con inquietudes geológicas, dado el carácter volcánico de las islas. El interés científico por estas islas también está vinculado al inicio del turismo. Viajeros extranjeros llegaban para sanar ciertas enfermedades como la tuberculosis dadas las benévolas condiciones climatológicas de las islas.

En el siglo XIX ${ }^{3}$, las Islas Canarias continúan siendo un lugar de interés científico. Aparte de Humboldt destacan en este contexto los trabajos de Leopold von Buch, Ernst Heinrich Philipp August Haeckel, Karl Wilhelm, Georg von Fritsch, Georg Hartung, Wilhelm Johann Reiß, Richard Greeff, Hermann Schacht, Friedrich Karl Noll, August Rothpletz y Hans Meyer. La lista de expediciones es interminable: el alemán al servicio de Rusia Georg von Langsdorff, su compatriota el naturalista Adalbert von Chamisso, el francés Luis Cordier, etc. Destaca la estancia durante largos años del francés Sabino Berthelot y la de dos años del botánico británico Philip Barker Webb, cuyas obras son decisivas para el conocimiento científico y etnográfico de las Islas Canarias. La geología tiene sus continuadores en la visita a Tenerife, Gran Canaria, La Palma y Lanzarote en 1853 del británico Charles Lyell y del alemán Karl von Fritsch en 1868 y del astrónomo escocés Charles Piazzi Smith en 1856.

Para elaborar esta información hemos consultado Hernández González (2011). 
En la segunda mitad del XIX despunta la antropología física y se pone en boga el evolucionismo ${ }^{4}$. El francés René Verneau y el italiano Paolo Mantegazza visitan las islas para excavar sus restos arqueológicos y buscar su pervivencia entre sus habitantes modernos. Las Islas Canarias llamaron también la atención de los científicos de esa época por el incremento del interés por el África negra. Así se comienza a estudiar la relación de las islas con África. La expansión imperialista británica por la India y la China llevó también a las Canarias a convertirse en escala de comerciantes y soldados que mostraron curiosidad por estas islas. A todos estos motivos, se une a la nueva moda de viajar entre las clases acomodadas. Se escriben las primeras guías turísticas sobre Canarias, como la de Alfred Samler Brown, que visitó las islas con ese objetivo entre 1889 y 1935. Sin embargo, el grueso de los viajeros lo constituye miembros de la élite británica con afecciones pulmonares atraídos por la benignidad del clima canario.

En cuanto a las obras científicas sobre Canarias escritas en el siglo XIX, sobresalen dos: la Histoire naturelle des Îles Canaries de Berthelot y Webb (1839) y la Physicalische Beschreibung der Canarischen Inseln de von Buch (1825). En la primera obra, estos dos científicos estudian de manera global la naturaleza insular, costumbres de las islas, cultura e idiosincrasia y un serio análisis del mundo aborigen a través de su Etnografía y Anales de la conquista de las Islas Canarias. En la segunda, von Buch describe la naturaleza geológica de las islas, su climatología y sus fuentes.

Es esta segunda obra, precisamente, la que hemos seleccionado para nuestro estudio. A continuación, la describimos y ponemos de manifiesto su gran importancia en el conocimiento natural de las Islas Canarias.

\section{Physicalische Beschreibung der Canarischen Inseln}

Tras su exploración con Christen Smith, siguiendo la costumbre de la época, Buch publica su propio relato de viaje en 1825, aunque Smith no hace lo propio con el suyo. Se trata de un manual de estudio para muchos científicos de la época con textos sobre flora aportados en su mayoría por Heinrich Friedrich Link ${ }^{5}$. Este libro tuvo gran repercusión en el ámbito de la geología de su tiempo y es sin duda el más importante sobre historia natural que se realizó sobre las Islas Canarias, y que, como obra de conjunto, no tuvo parangón en el siglo siguiente.

Physicalische Beschreibung der Canarischen Inseln se publicó primero en capítulos entre 1816 y 1820 y en 1825 sale en forma de libro. Está dividido en nueve capítulos: itinerario, clima, temperaturas de las fuentes y del suelo, medidas de las alturas de diversos puntos de las Islas Canarias, descripción de la isla de Tenerife, descripción de la isla de Gran Canaria, descripción de la isla de La Palma, descripción de la isla de Lanzarote y Fuerteventura, naturaleza de los fenómenos volcánicos en las Isla Canarias. Von Buch reproduce todos los itinerarios de sus excursiones por las diferentes islas y describe su geografía, sus fuentes, su orografía, sus pueblos, su entorno humano y sus paisajes (Relancio Menéndez, 2007: 128). Incluye registros de temperatura de distintos lugares, temperatura de fuentes, análisis de vientos, altitud de puntos estratégicos, etc. Ratifica su teoría de los cráteres de levantamiento con su descripción de las Cañadas (Tenerife), Tirajana (Gran Canaria), la

4 Von Buch realizó unos interesantísimos planteamientos predarwinistas en relación son su investigación en Canarias (Sarmiento Pérez, 2018).

5 Sobre el viaje de Link por España véase Link (2010). 
Caldera de Taburiente (La Palma), y con su descripción de la erupción de Fuencaliente en La Palma en 1677 y de Timanfaya en Lanzarote en 1730.

En general, podemos afirmar que es un libro importante en la literatura de viajes científicos a Canarias por las siguientes razones (Ibíd.: 125; Renn y Montesinos, 2002):

- El Archipiélago para él fue un destino científico exclusivo y no de paso hacia otro lugar como había sido el caso hasta entonces con otros científicos.

- Visita cuatro islas: Tenerife, Gran Canaria, La Palma y Lanzarote, y es el primero de los naturalistas europeos que realiza un estudio general de las tres últimas y uno exhaustivo de Tenerife, incluido su sur, y la primera descripción geológica de Lanzarote.

- Perfecciona la clasificación humboldtiana de los cinco pisos vegetales.

- Estudia la relación entre la climatología y las plantas, haciendo continuas mediciones de las temperaturas de las fuentes y de los suelos.

- Sus estudios geológicos serán fundamentales para el futuro de los saberes volcánicos sobre las Islas Canarias.

En resumen (Relancio Menéndez, 2007: 130), Physicalische Beschreibung der Canarischen Inseln fue un hito de primera magnitud en la exploración científica de estas islas y abrió camino a los estudios sistemáticos sobre Canarias en el siglo XIX.

\section{La traducción al español de Physicalische Beschreibung der Canarischen Inseln ${ }^{6}$}

Los traductores siempre hemos creído que lo ideal es traducir un texto desde la lengua meta sin pasar por una traducción intermedia a otra lengua. Sin embargo, este axioma no se cumple en la traducción de la literatura de viajes a Canarias escrita en alemán hasta 1914. Los viajeros decimonónicos tenían en cuenta la literatura anterior escrita sobre Canarias y se plagiaban unos a otros. La práctica más habitual era traducir las obras al francés y de esta traducción a otras lenguas, sin tener en cuenta la original (Montesinos Oltra, 2011:121). En ocasiones se traducía del español al francés o al alemán y de ahí, de nuevo al español. Es lo que se ha venido a denominar retrotraducción (Gambier, 1994: 413). Muchos autores no informan de sus fuentes originales y muchas, en todo caso, se han perdido.

La traducción de Physicalische Beschreibung der Canarischen Inseln no es una excepción. En 1836 C. Boulanger lo traduce al francés ${ }^{7}$ y de esta versión es de la que en 1999 el traductor José Antonio Delgado Luis efectúa la única traducción integral de la obra que existe al español. Resulta muy raro que una obra de tal repercusión en el ámbito geológico no gozara de una traducción hasta casi el final del siglo XX. Parte del libro, sin embargo, sí que había sido traducido antes al español ${ }^{8}$ y otra parte es una traducción del español al alemán

6 Para redactar este epígrafe se ha recurrido al capítulo de Batista Rodríguez et al. (2015).

$7 \quad$ Antes de él, Adrien de Jussieu efectúa una traducción parcial en francés (1833) que Boulanger utiliza.

8 Existen traducciones parciales de Hernández-Pacheco (2002[1909]: 244-246), Romero Ruiz (1991), Carracedo y Rodríguez Badiola (1991), Agustín Pallarés Padilla (2008). Tratamos estas traducciones en Monterde Rey y Sarmiento Pérez (2017). 
que efectuó von Buch y de ahí Boulanger lo tradujo al francés y Delgado Luis de nuevo al español. Veamos este último caso.

Al final de Physicalische Beschreibung der Canarischen Inseln existe una descripción de la erupción de Fuencaliente en La Palma en 1677 y de Timanfaya en Lanzarote en 1730. Esta parte de la obra fue escrita originalmente en español y el propio von Buch la tradujo al español. El texto de la erupción de La Palma era un manuscrito del licenciado don Juan Pinto de Guisla y el de Lanzarote, del párroco de Yaiza don Andrés Lorenzo Curbelo Perdomo (1744). Este último manuscrito contaba con una copia con la que se hace von Buch en Santa Cruz de Tenerife. Desafortunadamente, tanto el manuscrito original como sus copias han desaparecido, por lo que Delgado Luis tendría que haber traducido del texto francés sin remedio, de todas formas.

No ocurre lo mismo con la narración de la erupción de Fuencaliente que sepultó la Fuente Santa en La Palma. Sin embargo, Delgado Luis en vez de coger este manuscrito en español, lo traduce de la versión francesa de Boulanger al español. Por tanto, la cadena va del español al alemán, de ahí al francés y finalmente al español de nuevo. A pesar de no haber reproducido el texto original de de Guisla, según Batista Rodríguez et al. (2005: 114) el texto final resulta satisfactorio. Sin embargo, si Delgado Luis hubiera tomado el texto original habría evitado errores que efectuó von Buch, tales como sustituir términos típicos de aquella época por otros más familiares al traductor o fallos en la transcripción de ciertos topónimos como Fenianya por Teniquiga o Teneguía. De hecho, Pallarés Padilla (2007: 38) llega más lejos y afirma que existen numerosos fallos detectados, tanto en la traducción efectuada por von Buch, como en la francesa de Boulanger y la española de Delgado Luis. Afirma (Pallarés Padilla, 2008: 189-190) que von Buch hizo una traducción libre y descuidada, pues son numerosas las inexactitudes, tergiversaciones, alteraciones de nombres, cambios de fechas, etc. Además, von Buch intercala observaciones personales que, según Pallarés Padilla, son a veces poco acertadas y difíciles de deslindar del manuscrito original.

Por el contrario, según Batista Rodríguez et al. (Ibíd.), la traducción francesa de Boulanger es irreprochable. Además, es el propio von Buch quien revisa su traducción.

En cuanto a la traducción de Delgado Luis, en palabras de Batista Rodríguez et al. (Ibíd.: 119), es un trabajo minucioso y correcto, pero arrastra errores cometidos por von Buch en lo que se refiere a topónimos y fechas, que se podrían haber solventado con un trabajo de investigación. En el estudio terminológico que efectuamos más adelante, daremos cuenta de la adecuación de la traducción de los términos de la obra llevados a cabo por Delgado Luis. La situación de la traducción de Delgado Luis, a pesar de enmarcarse a finales del siglo XX, ha cambiado poco con respecto a las traducciones efectuadas en el siglo XIX: al igual que en esa época, traduce al español una obra traducida al francés. Si bien puede contar con más recursos a su alcance tales como diccionarios, bases de datos o acceso directo a especialistas.

Lorenzo Rodríguez copió los manuscritos de Juan Pinto de Guisla a finales del siglo XIX (1975) y los publica en sus Noticias para la historia de La Palma, gracias a lo cual no se ha perdido la obra original. Sin embargo, parece ser que Lorenzo Rodríguez desconocía que de Guisla era el autor del manuscrito original, aunque afirma que el texto procede del archivo del Sr. Marqués de Guisla Guiselin. 


\section{La traducción en España en los siglos XVIII-XIX ${ }^{9}$}

En el último cuarto del siglo XVIII comienza a tener fuerza la traducción de relatos modernos. Se trataba de obras narrativas relacionadas con el relato histórico o con un marcado componente educativo. Se traducían normalmente libros franceses o traducidos al francés. La traducción de las novelas inglesas también fue tardía, alrededor de la década de 1790, y también se tradujeron del francés y con un desfase de unos 50 años con respecto a la publicación del original. Destaca la traducción de obras religiosas desde el francés, italiano y latín y así como de libros de ámbito político, económico y filosófico que contribuyeron al desarrollo moderno del pensamiento económico y político en la España del siglo XVIII. Lo mismo ocurrió con la novela rusa: eran traducciones del francés. Esta tendencia solo cambia en el siglo XX.

Esta preferencia por el francés se debe a la galomanía que se implantó en esta época debida a la influencia de Francia en Europa en el ámbito cultural que se extendía a su lengua y su cultura. Además, en Inglaterra la actividad traductora era menor que en Francia, porque en París eran inferiores los costes de los materiales y la retribución de los traductores y porque había muchos más traductores de francés que de inglés. Las editoriales mantienen en nómina a los buenos traductores, aunque también existen traductores independientes que quieren firmar contratos directamente con los autores.

Como vamos a analizar la traducción de los términos de Physicalische Beschreibung der Canarischen Inseln, vamos a describir primero cómo se efectuaba esa traducción en las obras científicas traducidas en los siglos XVIII-XIX.

En estos siglos la traducción de la ciencia representa un porcentaje mucho menor que en otras áreas temáticas. Fernández Gómez y Nieto Fernández (1991) estudian las traducciones efectuadas desde el francés al español y encuentran que desde mediados del siglo XVIII hasta principios del XIX tan solo el 11,98\% de las obras traducidas pertenecen a un campo de la ciencia.

La iniciativa de las traducciones proviene en la mayor parte de los casos del propio traductor que suele ser especialista en la materia.

Montesinos Oltra (2011) analiza los prólogos de distintas traducciones de obras científicas del siglo XVIII. En ellos los traductores hablan de las dificultades de la traducción. En lo que respecta a los términos, en la mitad de los prólogos los traductores manifiestan que sienten la obligación de justificarse con respecto al uso de las voces especializadas. Incluso dos de los traductores consideran que los términos constituyen un sistema propio dentro de cada una de las ciencias, de manera que cada ciencia tiene su «idioma» particular. Encuentran dificultades en hallar las correspondencias castellanas de los términos extranjeros, sobre todo si el traductor no es especialista en la materia. La solución que proponen es consultar a especialistas en el campo científico del texto que se traduce.

Muchos traductores de los siglos XVIII y XIX muestran una preocupación lingüística por naturalizar los neologismos al castellano (Ibíd:: 144). Consideran imprescindible evitar la homonimia o la confusión con palabras existentes en castellano. Evitan emplear extranjerismos y favorecen los neologismos adecuados a la forma interna de la lengua castellana. Respecto a los términos que pudieran confundirse con alguna palabra castellana de la lengua común, proponen variar la terminación, siempre que no se pierda la transparencia

La información para este epígrafe se ha obtenido de Lafarga y Pegenaute (2004). 
semántica. Por último, coinciden en la necesidad de la univocidad de los términos para evitar confusiones. Hay que resaltar que como la mayoría de los traductores de estos siglos son especialistas en la materia científica que traducen, disminuye el «riesgo» de errores terminológicos en la búsqueda de equivalentes exactos.

Sin embargo, la traducción con la que nosotros vamos a trabajar data de 1999. En esta fecha están ampliamente superados los problemas de traducción científica que se podían plantear en el siglo XIX y así debería reflejarse en la búsqueda de equivalentes terminológicos.

\section{Terminología de la Descripción física de las Islas Canarias de Buch}

Como cualquier obra naturalista, la Physicalische Beschreibung der Canarischen Inseln cuenta con términos específicos en ese campo. Como ya hemos mencionado, el propósito de nuestro trabajo es analizar la terminología empleada en la única traducción de la obra completa que existe de esta obra al español realizada por José Antonio Delgado Luis (von Buch, 1999), estudio que nunca se ha llevado a cabo antes. Para ello, vamos a calcular la densidad terminológica; vamos a establecer qué métodos traductológicos se emplean en la búsqueda de equivalentes de los términos de la obra traducida al español y vamos a clasificar los términos según su formación. Empezaremos primero, sin embargo, describiendo los instrumentos de recogida de la muestra y presentando los términos aislados.

\subsection{Instrumentos de recogida de la muestra}

Para extraer los términos tanto de la obra original de von Buch en francés como de la traducida al español, hemos empleado el programa informático SDL MultiTerm Extract de Trados. Esta herramienta permite la extracción automática de terminología mediante una combinación de búsquedas de concordancia bilingüe y estadística (basada en la frecuencia terminológica) que muestra la aparición del término en contexto. Aún así, hemos hecho también una comprobación manual de la terminología extraída. Con los términos aislados, hemos creado una base de datos en el software MultiTerm de Trados. Cada ficha conceptual consta de los siguientes campos: término o términos en francés que representan el concepto; término o términos en español correspondientes a ese concepto; páginas en las que aparecen estos términos y números de recurrencias.

En la siguiente imagen podemos ver un ejemplo de ficha conceptual del concepto lapilli.

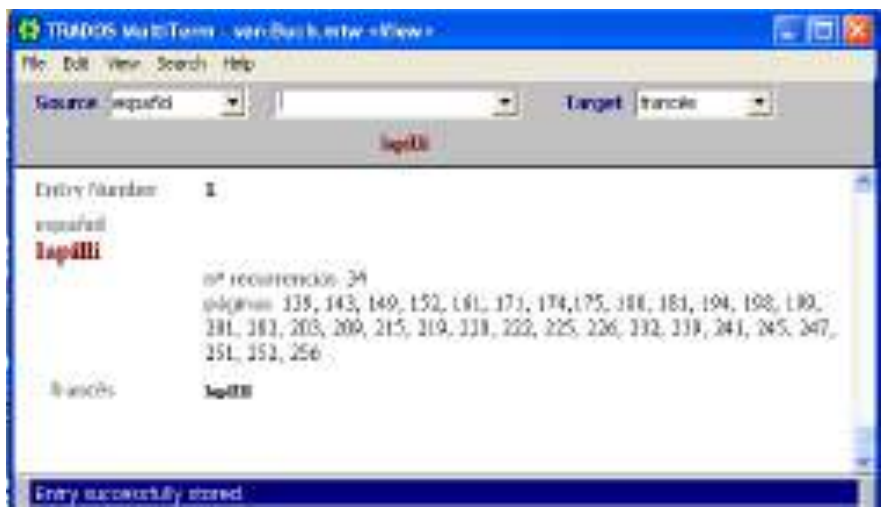

Figura 1. Ejemplo de ficha conceptual del concepto lapilli 


\subsection{La muestra}

Hemos hallado un total de 175 términos relativos a especies vegetales y término geológicos.

\subsubsection{Términos botánicos}

Existe un total de 96 términos del campo de la botánica. Todos ellos corresponden a nombres científicos en latín de especies vegetales que von Buch se encontró en su exploración de las islas Canarias. Términos tales como: Agave americana, Aira subspicata, Andianthum reniforme, Andromeda hynoides, Anethum faeniculum, Anthericum ossifragum, etc.

\subsubsection{Términos geológicos}

En total hemos hallado 79 términos geológicos diferentes. De estos términos cinco (6,32\%) representan conceptos relacionados con una sustancia química (ácido, ácido carbónico, vapor sulfúrico, vapor sulfuroso, anfígeno); dos (2,53\%), propiedades físicas (fiable, hemitropía); cinco pertenecen a distintos grupos $(6,32 \%)$ (fuente acídula, fumarola, hemitropo, mesotipo, sopladero); y los 67 restantes $(84,81 \%)$ son tipos de rocas y minerales (ej.: aragonito, traquita, feldespato, augita, hornablenda, basalto, toba, peridoto, etc.)

En el siguiente gráfico ilustramos el porcentaje de términos geológicos aislados.

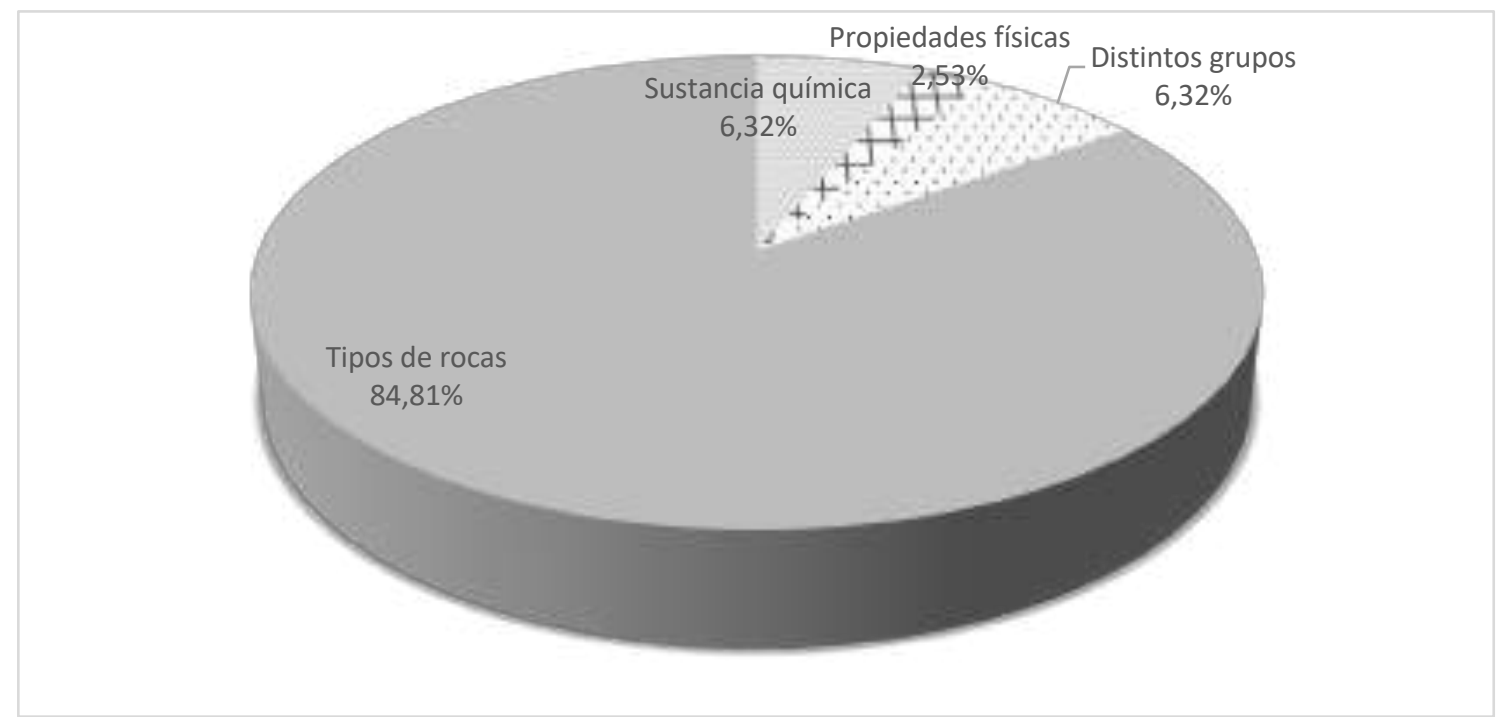

Gráfico 1. Tipos de términos geológicos en la obra de von Buch

\subsection{Densidad terminológica}

Lo primero que llama la atención, es el bajo porcentaje de términos que existe en esta obra. Solo un 3,8 \% de las denominaciones son términos. Según este porcentaje, el escrito no podría considerarse de carácter especializado, sino semi-especializado, ya que para ello la densidad terminológica debería ser de al menos un 15\% (Arntz y Picht, 1995:48). 


\subsection{Métodos de traducción de términos}

Hemos seleccionado la clasificación de Molina y Hurtado (2002: 499-500), clasificación que se ha empleado con éxito en trabajos de análisis terminográfico (Lozano y Matemala, 2009). De todos sus métodos hemos aislado solo los que tienen relación con la búsqueda de equivalentes de términos: adaptación, amplificación, calco, compensación, descripción, equivalente exacto, reducción, generalización, particularización, préstamo y traducción literal.

La adaptación consiste en remplazar elementos culturales de la lengua origen (LO) por elementos culturales de la lengua meta (LM). Ejemplo: cycling para el francés y cricket para el inglés.

La amplificación añade detalles que no existían en el texto origen (TO). Ejemplo: traducir He talked himself out of a job (inglés) por Il a perdu sa chance pour avoir trop parlé (francés).

El calco es la traducción literal de una palabra. Ejemplo: air conditioning (inglés), aire acondicionado (español).

La compensación consiste en introducir un elemento, una información o un efecto estilístico en otro lugar del texto, porque no tiene cabida en el mismo lugar que en el TO. Ejemplo: traducir I was seeking thee, Flathead (inglés) por En vérité, c'est bien toi que je cherche, O Tête-Plate (francés).

La descripción es la sustitución de un término o expresión por una descripción. Ejemplo: airbag (inglés) y neumático amortiguador de golpes automovilístico (español).

El equivalente exacto es el uso de un término o expresión reconocido como equivalente en la LM. Ejemplo: chair (inglés) y silla (español).

La reducción consiste en sintetizar elementos lingüísticos del TO. Ejemplo: the month of fasting (inglés) en oposición a Ramadan cuando se traduce al árabe.

La generalización consiste en añadir elementos lingüísticos. Ejemplo: guichet, fenêtre, devanture (francés) y window (inglés).

La particularización es el uso de un término más preciso o concreto. Ejemplo: window (inglés) y guichet, fenêtre, devanture (francés).

El préstamo es el empleo de una denominación extranjera. Ejemplo: pub (español).

La traducción literal consiste en traducir palabra por palabra una expresión o frase. Ejemplo: traducir They are as like as two peas as (inglés) por Se parecen como dos guisantes (español).

Veamos qué métodos sigue la terminología de Physicalische Beschreibung der Canarischen Inseln. 


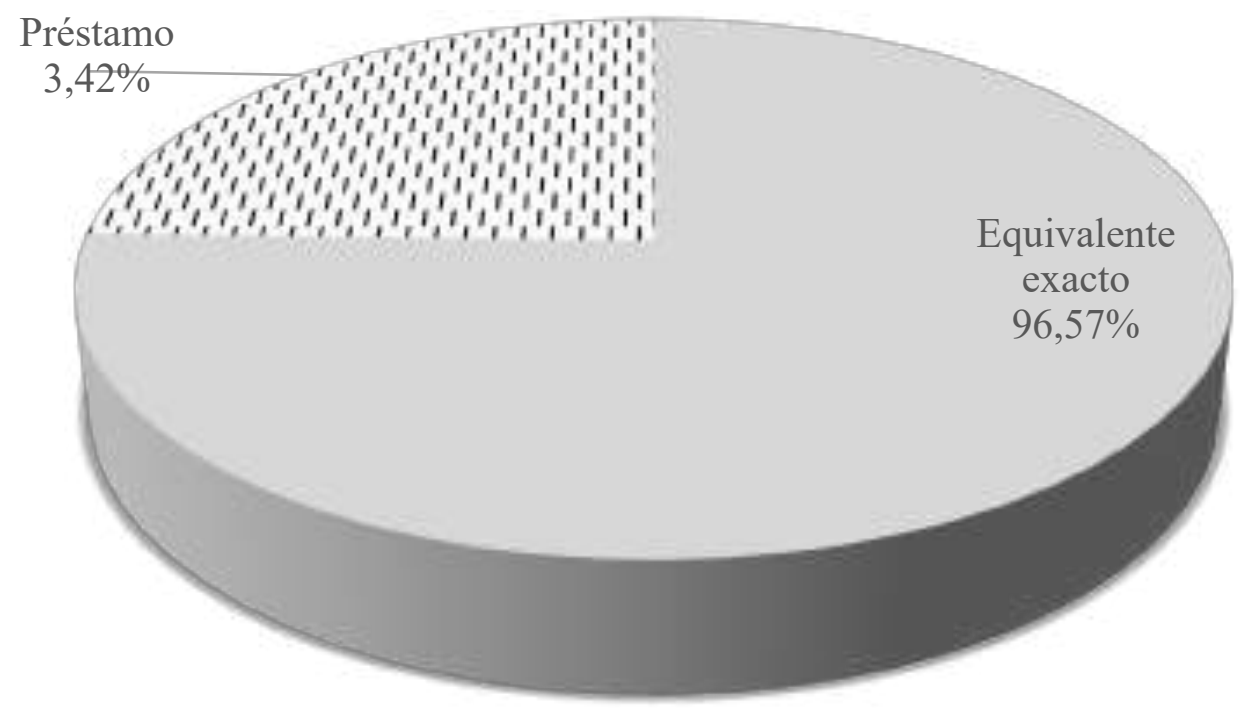

Gráfico 2. Métodos de traducción de términos

Como vemos en el gráfico anterior, de los términos aislados 169 (73 términos geológicos y todos los 96 términos botánicos) (96,57\%) se han traducido buscando el equivalente exacto y seis $(3,42 \%)$ mediante un préstamo. Se trata de los siguientes términos pertenecientes a nombres de rocas: gneis, ladrillo de Engers, pechstein, roca de Wolkenburg, toba de Pausilippe, wacke. A pesar de haberse empleado un préstamo, son, en realidad, los equivalentes exactos en español ${ }^{10}$ excepto toba de Pausilippe, pues no existe otro equivalente en nuestra lengua. En el caso de toba de Pausilippe, se trata de un calco del francés. Pausilippe es un barrio residencial en las colinas de la ciudad de Nápoles que en español se conoce como Posillipo. Este error concuerda con lo que habían denunciado otros autores sobre los fallos que existían en esta obra en cuanto a los topónimos empezando ya por el original en alemán (Pallarés Padilla, 2008: 189-190).

En cuanto al resto de los préstamos, Engers ${ }^{11}$ y Wolkenburg ${ }^{12}$ son topónimos y, por tanto, no se traducen. Wacke es una roca basáltica cuyo nombre procede del inglés y se ha adoptado en español. Pechstein es el término procedente del alemán con el que se denomina en español a la obsidiana con mayor de 3-44\% de peso en agua. Finalmente, en cuanto a gneis, también es un término alemán que se emplea en castellano. Su etimología no está clara. Según el Aschehoug og Gyldendals Store norske leksikon (Henriksen, 2007) procede del verbo del medio alto alemán gneist (chispear), puesto que el gneis es una roca metamórfica que produce brillos al ser percutida.

En resumen, podemos afirmar que se han empleado los equivalentes exactos de todos los términos del francés al español exceptuando uno: toba de Pausilippe.

\subsection{Métodos de formación de términos}

10 Para verificar la traducción de los términos en español, hemos contado con la ayuda del Dr. Fernando Hernández García, geólogo especializado en Canarias.

11 Distrito de Neuwied en el flanco derecho del río Rhin en Alemania localizado cerca de Koblenz.

12 Salón de eventos de Colonia (Alemania). 
En relación con el texto que nos ocupa, nos referiremos ahora los principales recursos para la formación de términos. Los lenguajes especializados cuentan con cinco recursos para la formación de términos (Arntz y Picht, 1995: 147): terminologización, composición, derivación, conversión, calco léxico y abreviación.

La terminologización se produce cuando una palabra de la lengua común es tomada por un lenguaje especializado y allí adopta y contenido especializado. Por Ejemplo: gato que en la lengua común es un animal doméstico, en mecánica es el dispositivo que eleva el coche.

La composición es el proceso por el que «términos identificables para el locutor se conjuntan en una unidad nueva de significado único y constante» (Beneviste, 1977: 172). Un ejemplo sería válvula de descarga. Las investigaciones de Goffin (1992: 431) indican que el 85\% de la terminología está representado por más de un elemento; esto es, los términos están formados por la unión de dos o más vocablos. Este método es, junto con la sufijación, el método más común de formación de nuevos términos en español (Almela, 1999: 120).

La derivación consiste en formar términos añadiendo un prefijo (prefijación) o un sufijo (sufijación) a una denominación existente. Ejemplo: postparto.

La conversión es el método de formación de términos por el que una nueva denominación se crea mediante el cambio de categoría léxica. Ejemplo: el adjetivo constante pasa a ser el sustantivo la constante en las fórmulas matemáticas.

El calco léxico es la traducción literal palabra por palabra de un término extranjero. Ej.: traduction assistée par ordinateur es traducido como traducción asistida por ordenador.

Por último, con el método de abreviación se toma un término y se reduce gráficamente (ej.: pág. por página) o se toman sus letras iniciales (TDF por toma de fuerza) para crear un nuevo término.

En nuestra muestra, 132 términos (36 términos geológicos y todos los 96 términos botánicos) $(75,42 \%)$ se han creado por composición (ácido carbónico, cristal de analcima, conglomerado basáltico, etc.). Las 43 denominaciones restantes $(24,57 \%)$ son denominaciones simples (formadas por un solo término) de nueva formación que no sigue ninguno de los métodos de formación de nuevos términos.

Como vemos, en nuestra muestra no se cumple la investigación de Goffin (1992), pues el método de composición no llega al $85 \%$ que él había comprobado.

A continuación, veamos un gráfico ilustrativo de los métodos de formación de términos empleados. 


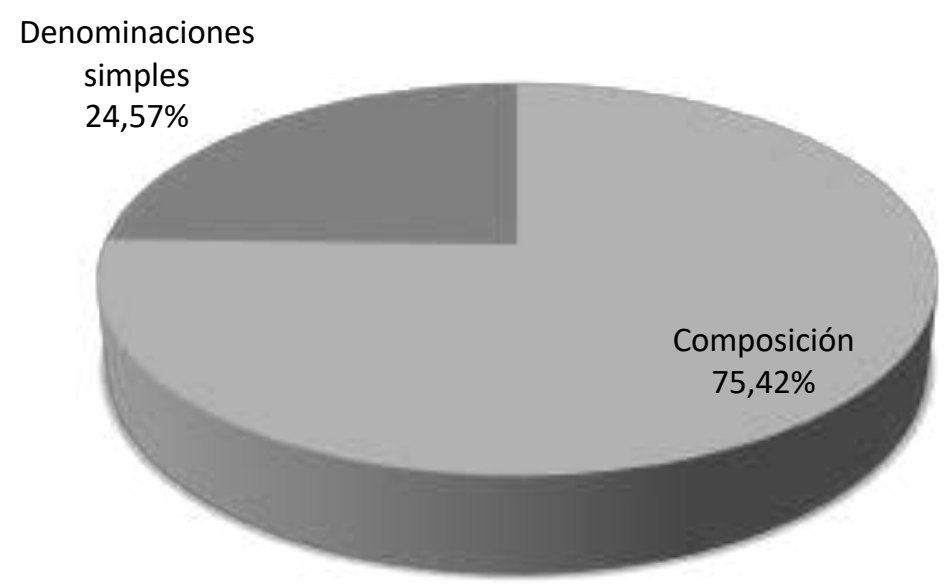

Gráfico 3. Métodos de formación de términos

\section{Conclusiones}

Physicalische Beschreibung der Canarischen Inseln de Leopold fue el libro de mayor repercusión en el campo de la geología que se escribió sobre Canarias en el siglo XIX. Fue escrito por von Buch, el más prestigioso geólogo de su época. A pesar de su gran importancia, no se tradujo en su totalidad al español hasta 1999 y esta versión la efectuó Delgado Luis a través de la traducción de esta obra al francés de Boulanger en 1836. La traducción al español a partir de otra traducción al francés era una práctica habitual en el siglo XIX. Los capítulos del libro referente a erupciones volcánicas en La Palma y Lanzarote provienen de traducciones que efectuó von Buch de manuscritos de autores españoles. El texto original de Lanzarote se ha perdido, no así el de La Palma. Sin embargo, en la versión española, en lugar de tener en cuenta los manuscritos originales, se ha traducido del francés. En general, a pesar de errores en topónimos y fechas, se puede afirmar que la traducción al español es minuciosa y satisfactoria (Batista Rodríguez et al. 2005: 114), aunque haya autores que no compartan esta afirmación (Pallarés Padilla, 2008: 189-190) por los fallos antes mencionados.

A pesar de la gran repercusión científica de Physicalische Beschreibung der Canarischen Inseln nadie hasta ahora ha analizado su terminología, por lo que nos pareció interesante efectuar este estudio en su traducción al castellano. Hemos comprobado que, aunque se trata de un libro científico, su densidad terminológica es baja, solo 3,8\% de los vocablos son términos (175 términos). Este bajo porcentaje quizá se deba al hecho de que en la época de von Buch la Geología estaba todavía en un estado muy incipiente. Posteriormente, por ejemplo, con Charles Lyell (1797-1875) se fueron introduciendo nuevos conceptos geológicos.

De los 79 términos geológicos aislados, cinco $(6,32 \%)$ representan conceptos relacionados con una sustancia química; dos (2,53\%), propiedades físicas; cinco pertenecen a distintos grupos $(6,32 \%)$; y los 67 restantes $(84,81 \%)$ son tipos de rocas y minerales. Además, se han hallado 96 términos relativos a nombres científicos de plantas. Un $96,57 \%$ de los términos se ha traducido mediante un equivalente exacto y 3,42\% mediante un préstamo, aunque en el fondo se tratan también de equivalentes exactos, pues son las denominaciones que se emplean en castellano. De todos los términos traducidos tan solo uno no corresponde realmente a una denominación empleada en castellano. Se trata de toba de Pausilippe que debería haberse traducido por toba de Posillipo. 
Un $24,57 \%$ de los términos son denominaciones simples, mientras que un $75,42 \%$ se han creado por composición.

Podemos concluir que desde el punto de vista terminológico Physicalische Beschreibung der Canarischen Inseln es una obra con muy baja densidad terminológica y que su traducción al español destaca por la fiabilidad de las denominaciones terminológicas en castellano representado por el alto porcentaje de equivalentes exactos. Con este estudio esperamos haber aportado una nueva dimensión al conocimiento de esta importante obra cuya terminología estaba sin estudiar.

\section{Declaration of conflicting interests}

The author(s) declared no potential conflicts of interest with respect to the research, authorship, and/or publication of this article.

\section{Funding}

The author(s) received no financial support for the research, authorship, and/or publication of this article.

\section{Sobre la autora}

La Doctora Ana María Monterde Rey es actualmente Profesora Titular de la Universidad de Las Palmas de Gran Canaria y trabaja en esta institución como docente de Terminología y Documentación Aplicada a la Traducción desde el año 1995. Sus líneas de investigación son la terminografía informatizada, la representación conceptual y los lenguajes para fines específicos en especial el español de negocios.

Ha publicado dos libros para la docencia de la Terminología en la Licenciatura de Traducción e Interpretación (Curso de introducción a la terminología para traductores e intérpretes, 1998; Ejercicios de introducción a la terminología para traductores e intérpretes, 2002), así como diversos capítulos de libro y artículos.

\section{Agradecimientos}

Al Doctor Marcos Sarmiento Pérez por sus consejos y apoyo en el proceso de redacción de este texto.

\section{Bibliografía}

Almela Pérez, R. (1999) Procedimientos de formación de palabras en español. Barcelona: Ariel

Arntz, R.; Picht, H. (1995) Introducción a la Terminología. Madrid: Fundación Germán Sánchez Ruipérez. 
Batista Rodríguez, J.J.; Tabares Plasencia, E.; Hernández Socas, E. (2015). Alcance y límites de la documentación histórica para traducir literatura de viajes sobre Canarias (El caso de los viajaros decimonónicos de lengua alemana). En Marcelo Wirnitzer, G. (coord.). Traducir la historia desde diferentes primas (pp. 99-176). Las Palmas de G.C.: Servicio de Publicaciones y Difusión Científica de la Universidad de Las Palmas de G.C.

Beneviste, E. (1977) Problemas de lingüística general II. Madrid: Siglo XXI.

Boulanger, C.-L. (1844). Carte géologique, minéralurgique et topographique du Département de l'Allier. Paris: Moulins.

Boulanger, C.-L. (1849). Carte géologique du Département du Cher. Paris: Corps des Mines.

Boulanger, C.-L. (1850). Texte explicatif de la carte géologique du Département du Cher. Paris: Imprimerie nationale.

Buch, L. von (1819a). Allegemeine Übersicht der Flora auf den Canarishen Inseln. In Abhandllungen der Königlichen Akademie der Wissenschaften in Berlin (pp. 337-384). Berlin.

Buch, L. von (1819b). Über einen vulcanishen Ausbruch auf der Insel Lanzerote [sic.]. In Abhandllungen der Königlichen Akademie der Wissenschaften in Berlin (pp. 69-82). Berlin.

Buch, L. von (1825). Physicalische Beschreibung der Canarischen Inseln. Berlin.

Buch, L. von (1836). Description physique des Îles Canaries. (Traducción: C. Boulanger). Paris: Levrault Libraire.

Buch, L. von (1999). Descripción física de las Islas Canarias. (Estudio crítico: Manuel Hernández González. Traducción: José A. Delgado Luis). Santa Cruz de Tenerife, JADL.

Carracedo, J.C.; Rodríguez Badiola, E. (1991). Lanzarote: la erupción de 1730. Arrecife: Cabildo Insular de Lanzarote.

Curbelo Perdomo, A.L. (1744). Diario de apuntaciones de las circunstancias que acaecieron en Lanzarote, cuando ardieron los volcanes, año de 1730, hasta 1736, dedicado al ilustrísimo señor don Juan Francisco Guillén, Obispo de Canarias, en 1744. [Inédito].

Fernández Gómez, J.F. y Nieto Fernández, N. (1991). Tendencias de la traducción de obras francesas en el siglo XVIII. En Donaire, M.L. y Lafarga, F. (eds.) Traducción y adaptación cultural: España-Francia (pp. 579-591). Oviedo: Universidad de Oviedo.

Gambier, Y. (1994). La retrotraduction, retour et détour. Meta: journal des traducteurs / Meta: Translators' Journal, 39(3), $413-417$.

Goffin, R. (1992) Du synthème au phraseolexème en terminologie diffèrentielle. En Terminologie et Traduction , 2-3, 431-438. Paris: Comisión de las Comunidades Europeas.

Henriksen, P. (2007) Aschehoug og Gyldendals Store norske leksikon. <https://snl.no/> [28/01/2018]

Hernández, M. (2011). Viajes científicos en Canarias. Museos de Tenerife. <https://bit.ly/2cqMl2E> [16/02/2018]

Hernández-Pacheco, E. (2002[1909]). Por los campos de lava. Relatos de una expedición científica a Lanzarote y a las Isletas canaria. Descripción e historia geológica (1907-1908). Teguise: Fundación César Manrique.

Jussieu, A. (1833). Coup d'oeil sur la flore des Îles Canaries. Archives de Botanique (pp. 289-316) 〈https://bit.ly/2E63azJ> [16/02/2018].

Lafarga, F.; Pegenaute, L. (eds.) (2004). Historia de la traducción en España. Salamanca: Ambos mundos.

Link, H.F. (2010). Viaje por España. Edición e introducción de Sandra Rebok y Miguel Ángel Puig-Samper. Traducción de Marta Fernández Bueno. Madrid. Consejo Superior de Investigaciones Científicas.

Lorenzo Rodríguez, J.B. (1975[fines s. XIX]). Noticias para la historia de La Palma. La Laguna/ Santa Cruz de La Palma: CSIC/ Centro de Estudios Canarios/ Cabildo Insular de La Palma, tomo I.

Lozano, D./ Matamala, A (2009). The translation of medical terminology in TV fiction series: the Spanish dubbing of E.R. VIAL, 6, 73-87.

Molina, L. and Hurtado, A. (2002). Translation Techniques Revised: A dynamic and functionalist approach. Meta, 47(4), 498-512.

Monterde Rey, A. M. y Sarmiento Pérez, M. (2017). Sobre la traducción al español de la primera descripción geológica del Archipiélado Canario: Physicalische Beschreibung der Canarischen Inseln”. En Sarmiento Pérez, M. (ed.). Historia de la traducción y de la interpretación en Canarias (pp. 59-86). Madrid: Síntesis. 
Montesinos Oltra, A. (2011). La traducción científica en España en el siglo XVIII. En Estudio de la versión española de L’Histoire Naturelle de Buffon (1785-1805) por J. Clavijo y Fajardo. Tesis doctoral dirigida por Brigitte Lépinette Lepers. Valencia: Universidad de Valencia.

OCLC (2016). WorldCat Identities. <https://bit.ly/2xmNEti> [28/01/2018]

Oliver Frade, J. M.; Relancio Menéndez, A. (2007). El descubrimiento de las Islas Canarias. Santa Cruz de Tenerife: Fundación Canaria Orotava de Historia de la Ciencia.

Pallarés Padilla, A. (2007). Nuevas aportaciones al conocimiento de la erupción de Timanfaya (Lanzarote). Discursos académicos de la Academia de Ciencias e Ingenierías de Lanzarote, Arrecife.

Pallarés Padilla, A. (2008). Consideraciones en torno al manuscrito del cura de Yaiza, Andrés Lorenzo Curbelo, sobre las erupciones volcánicas del siglo XVIII en Lanzarote. En: XII Jornadas de Estudios sobre Lanzarote y Fuerteventura (pp. 187-201). <https://bit.ly/2E2DCmS> [12/02/2018]

Renn, J.; Montesinos, J. (2002). Expediciones científicas a las Islas Canarias en el periodo romántico (1770-1830). Fundación Canaria Orotava de Historia de la Ciencia. Proyecto Humbold. <https://bit.ly/2OOHoRq>_[28/01/2018]

Romero Ruiz, C. (1991). Las manifestaciones volcánicas del Archipiélago Canario. Santa Cruz de Tenerife: Consejería de Política Territorial del Gobierno de Canarias, 3 tomos.

Sarmiento Pérez, M. (2018). Planteamiento predarwinista de Leopold von Buch. En: Vallejo G. et al. (eds.) Darwin y el Darwinismo desde el sur del sur (pp. 99-110). Madrid: Ediciones Doce Calles.

Treccani (2016). Enciclopedia de la cultura italiana. < https://bit.ly/2OFu56j> [16/03/2018]

Webb, P.B.; Berthelot, S. (1839). Histoire naturelle des Îles Canaries. Paris: Béthune. 